\title{
GCU
}

Glasgow Caledonian

University

University for the Common Good

\section{An economical and feasible teaching tool for biomedical education}

\author{
Karadaglic, Dejan; Stojanovic, Radovan
}

Published in:

24th International Symposium on Computer-Based Medical Systems (CBMS), 2011

DOI:

10.1109/CBMS.2011.5999116

Publication date:

2011

Document Version

Author accepted manuscript

Link to publication in ResearchOnline

Citation for published version (Harvard):

Karadaglic, D \& Stojanovic, R 2011, An economical and feasible teaching tool for biomedical education. in 24th International Symposium on Computer-Based Medical Systems (CBMS), 2011 . IEEE, pp. 1-5, 24th International Symposium on Computer-Based Medical Systems (CBMS), 2011, Bristol, United Kingdom, 27/06/11. https://doi.org/10.1109/CBMS.2011.5999116

\section{General rights}

Copyright and moral rights for the publications made accessible in the public portal are retained by the authors and/or other copyright owners and it is a condition of accessing publications that users recognise and abide by the legal requirements associated with these rights.

Take down policy

If you believe that this document breaches copyright please view our takedown policy at https://edshare.gcu.ac.uk/id/eprint/5179 for details of how to contact us. 


\title{
An economical and feasible teaching tool for biomedical education
}

\author{
Radovan Stojanović \\ University of Montenegro, Montenegro \\ stox@ac.me \\ Dejan Karadaglić \\ University of Manchester, UK \\ dejan.karadaglic@manchester.ac.uk
}

\begin{abstract}
There are many biomedical instruments around, and they have addressed a broad variety of clinical and technical issues. However, there is still a room for their improvement in terms of price, portability, signal to noise ratio, multi-channel work, universal and simple connectivity, on-line processing and running under standard software, user-friendliness. A real-time prototype system has been developed that attempts to address some of these limitations. It includes simple circuits for signal detection and modulation, microphone input and sound card for data acquisition and MATLAB software for demodulation, processing, viewing and data handling. In this paper we are concerned with physiological signals like electrocardiograph (ECG) and photoplethysmograph $(P P G)$ and focus on development of effective laboratory teaching tools for the purpose of biomedical engineering education. The same principle can be applied to some other courses, as well.
\end{abstract}

\section{Introduction}

The most commonly available tools for laboratory examples typically have the sensors which acquire the observed signals and transfer them to a computer using the data acquisition systems in form of DAQ cards or external portable devices. The DAQ cards make the system bulky and expensive. The portable devices are better solution since they use standard ports like serial (COM), parallel (LPT) or USB. Both approaches require special software drivers, which are usually not freely available and differ for one manufacturer to the other. On the other hand, the most of today desktop or laptop computers do not even have the parallel or serial ports, while the programming of USB port is not trivial task and some standard computation software like MATLAB do not support this interface yet.
Microphone input (MIC) is the only interface built in almost all computers, regardless they are desktop or laptop, and even can be found in mobile phones or personal digital assistants (PDAs). An alternate data transfer method using this type of interface proves out to be very convenient and effective since the integrated sound cards allow acquisition process at high resolution (up to 24bits) and adjustable sampling rate (up to $44 \mathrm{KHz}$ ) behind built-in amplifiers, analog switchers and filters. Additionally, the sound cards can be easily controlled from a broad range of standard applications.

However, the sound card filters are of band pass nature with $20 \mathrm{~Hz}-20 \mathrm{kHz}$ range, both on the input and the output. Most commonly, down border frequency varies from $50 \mathrm{~Hz}-200 \mathrm{~Hz}$ depending on the quality of the device. It means that the DC and slow varying signals cannot be faithfully reproduced by direct coupling to the microphone input. The most of the information with the physiological signal, like ECG and PPG, are just below $20 \mathrm{~Hz}$ and these signals should be considered as low frequency or even DC.

A way to circumvent this limitation is to use low frequency signal to modulate a carrier in the audio range; capture the modulated signal using a soundcard; and then demodulate in software [1]. MATLAB provides ideal solution for such scenario since it has functions for easy control of sound cards as well as functions for demodulation, filtering and powerful signal processing, data analyze and visualization.

In the presented work we propose the laboratory toolset for physiological measurements based on suggested approach, which we believe can be widely 
applied and accepted from students, teachers and researchers.

\section{Methodology}

The laboratory toolset we propose is quit modular and consists of both, hardware and software units. Its final appearance, now in the process of exploitation and full use, is shown in Fig 1.

Students begin the experimental sessions by designing sensing amplifiers and filters on experimental board. They use standard operation amplifiers (for PPG) and integrated instrumentation amplifiers (for ECG). The attention has been given to design issues like: type of electrodes, contact effects, amplitude of the input signals, noise influences and reductions, the span of the output signal, power consumption, power supplying methods (single or double) etc. During circuit composing the students are checking and observing the signals by oscilloscope in different test points (before filtering, after filtering, $\mathrm{DC}, \mathrm{AC}$ level etc). In our case we use ground-free electrodes and ECG signal is taken from fingers that is more convenient way for student exercises.

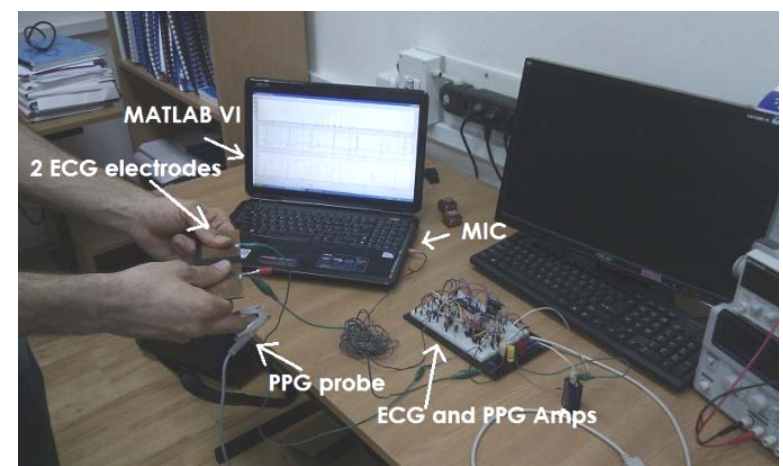

Figure 1. Laboratory toolset interfacing to mic. input

After amplification and hardware filtering the ECG and PPG signals amplitude modulate square wave carriers at frequencies of $2 \mathrm{KHz}$ and $4 \mathrm{KHz}$, Fig. 2. Pulse amplitude single polarity modulation is used. Two modulated signals are then mixed by summing circuit and sent to the microphone input.

On the host side MATLAB software performs the control and acquisition of the sound card, filtering of mixed modulated signal in order to separate carriers, demodulation in parallel branches and low-pass filtering of demodulated PPG and ECG signals, Fig. 3. In demodulation process $44.1 \mathrm{Ksamples}$ are acquired per second and stored in temporary array which is then being separately filtered and demodulated using envelope detection method based on Hilbert transform. Array is then fed to a 5 seconds long buffer and plotted to the screen.

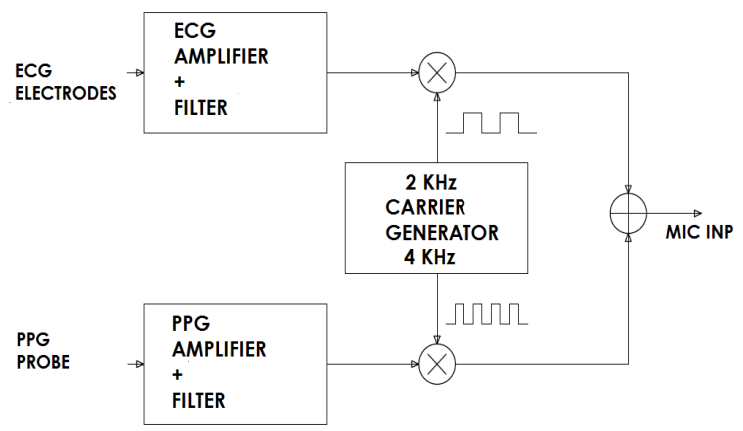

Figure 2. Hardware architecture of front end.

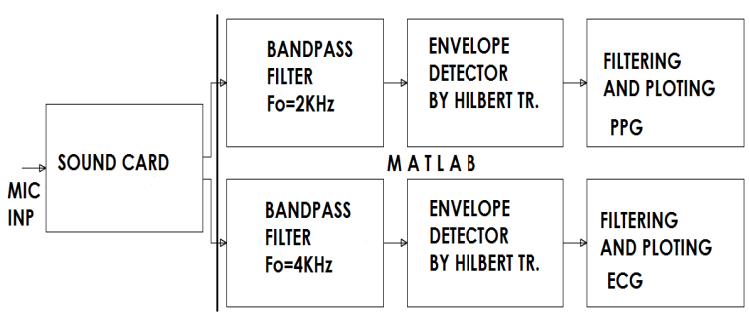

Figure 3. Architecture of software unit.

Overall MATLAB software is integrated in a Virtual Instrument (VI), the screenshot of which is shown in Fig. 4. Uppermost diagram shows mixed modulated signal with $2 \mathrm{KHz}$ and $4 \mathrm{KHz}$ carriers, while the lower two illustrate demodulated signals ECG and PPG. In addition to basic functions for demodulation and filtering, the VI performs data analyze in time and frequency domain as well as QRS detection and Heart Rate Variability (HRV). The selection between frequency and time mode is enabled by check box options.

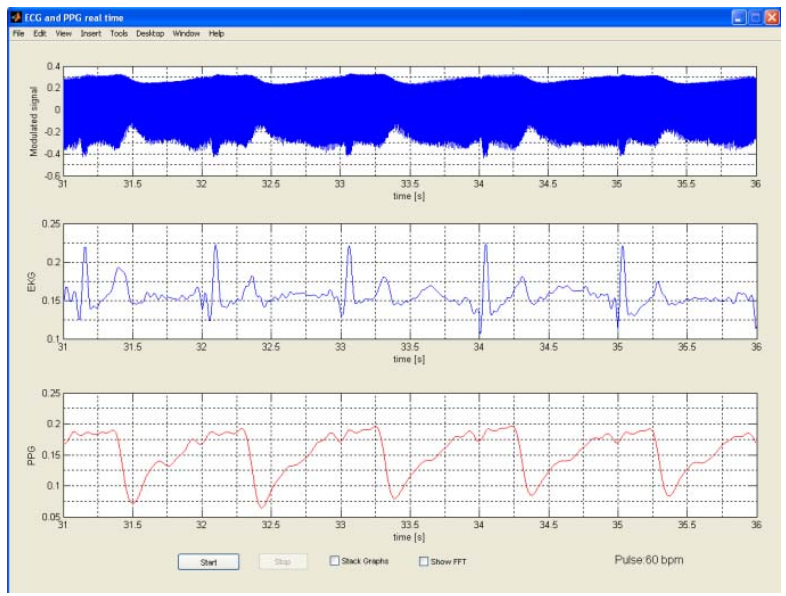

Figure 4. Screenshot of VI. 


\section{Example of exercises}

During student exercises, due to its simplicity, easy handling and speed, the ECG signal is taken from fingertips (see Fig 1) in two leads, ground free configuration. It can also be done in chest version of LEAD 2 or RA-LA-RL. The PPG signal is taken from finger.

For several exercises students connect the same electronic circuit [2]. It consists of PPG and ECG amplifiers, filters and MC unit. The ECG amplifier is based on INA 121 (BURR-BROWN) circuit with low power consumption $(<0.5 \mathrm{~mA})$ and high input impedance (FET's inputs). PPG amplifier is, also, designed by using standard components (quad operation amplifier LM 324), while as a sensor Nelcor DS-100A finger configuration was used. Main filtering processing task is realized by software, while the hardware filters are reduced to simple configurations of the first order filters (LP, HP and BP). All circuits are powered by single supply $+5 \mathrm{~V}$ in order to ensure compatibility with A/D span. The carrier signals of square wave type are generated by microcontroller (ATMEGA16) and pulse amplitude modulation is performed by bilateral analog switch. The modulated signals with $2 \mathrm{KHz}$ and $4 \mathrm{KHz}$ carriers are mixed using simple summing circuit and forward to standard microphone input.

\subsection{Processing ECG and PPG signal in time domain and detecting PTT}

The heart is a hollow, muscular organ which through a coordinated muscle contraction generates the force to circulate blood throughout the body. Each beat of our heart is triggered by an electrical impulse from special sinus node cells in the right upper chamber of our heart. The electrical impulse travels to other parts of the heart and causes the heart to contract. An ECG records these electrical signals. Looking at the ECG you can see that there is a recurrent pattern in this signal. Every characteristic of this pattern has certain features and a certain physiological meaning.

PPG is a non-invasive method to detect cardiovascular pulse wave that propagates through the body using a light source and a detector. Simply we graphically illustrate the blood circulation through our finger or ear lobe.

Pulse Transmit Time (PTT) is the time between the heartbeat and the arrival of the blood pressure wave to a peripheral site. To calculate it we need simultaneously to acquire ECG and PPG signals. Then the PTT is calculated as a time interval between R peak of the EKG and a point corresponding to maximum or minimum of PPG signal.

During this exercise the students become familiar with ECG and PPG signals and PTT. ECG and PPG are simultaneously sensed (like in Fig 1.), amplified, pre-filtered and modulated. Modulated signal, Fig. 5 (up), is acquired by sound card in rate 44.1Ksamples/sec and filtered in parallel branches by ideal band bass filters, $2 \mathrm{KHz} \pm 30 \mathrm{~Hz}$ and $4 \mathrm{KHz} \pm 30 \mathrm{~Hz}$, in order to separate carriers with PPG and ECG. Thereafter, the separated carriers are passed through demodulator block based on Hilbert transformation. The demodulated signals consists noise and should be re-filtered by low pass filters with border frequencies of $10 \mathrm{~Hz}$ (PPG) and $30 \mathrm{~Hz}$ (ECG). The filtered ECG and PPG signals are plotted together or separately, Fig. 5 (down).

By displaying ECG and PPG signals in the same window, and using built-in "cursor function" the PTT can be measured as distance between R peak and local minimum of PPG signal, Fig. 5. There are mathematical relations between PTT and systolic and diastolic pressures (SBP, DBP) that gives us a possibility for cuffless blood pressure monitoring [3].

The students record their ECG and PPG signals and determine the PTT. Simultaneously they measure BP with digital meter during the rest and physical exercise noticing that shorter PTT corresponds to higher BP and vice versa. At the end, they print their waves on paper and analyze them taking into account the theoretical knowledge.
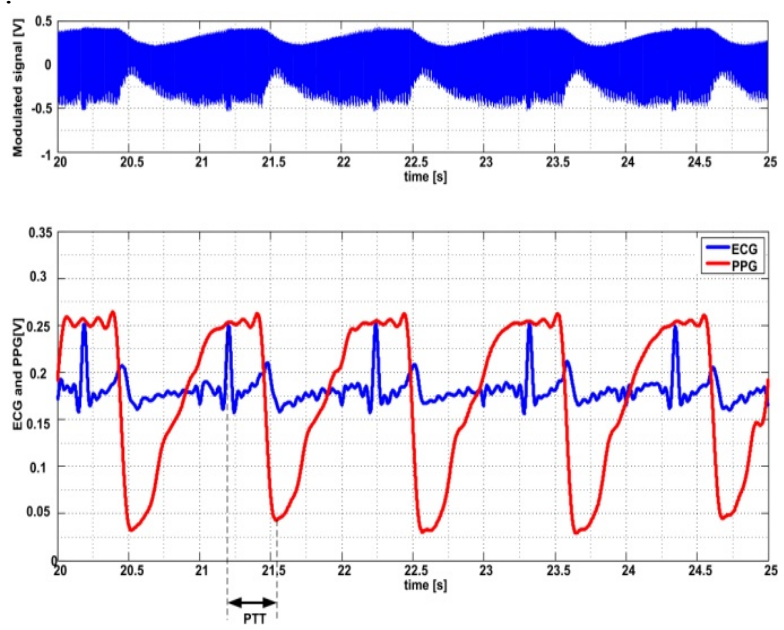

Figure 5. Mixed modulated signal and demodulated PPG and ECG signals, time domain. 


\subsection{Processing ECG and PPG signal in frequency domain and detecting $H R$}

Heart rate (HR) is the number of heartbeats per unit of time, typically expressed as beats per minute (bpm). The measurement of HR is used by medical professionals to assist in the diagnosis and tracking of medical conditions. It is also used by individuals who are interested in monitoring their heart. The RR interval is the inverse of the HR.

During this exercise the students observe the ECG and PPG signals in frequency domain. The MATLAB Fast Fourier Transform (FFT) functions are applied to modulated and demodulated signals, Fig. 6. First diagram show the spectrum of mixed modulated PPG and ECG signals. Two peaks on $2 \mathrm{KHz}$ and $4 \mathrm{KHz}$ correspond to the carrier frequencies. Around carriers are frequency components produced by the modulation signals. The students note that the spectrum around ECG carrier is wider and "richer" in context of harmonics than one around PPG. After the separation, demodulation and low pass filtering, the FFT is applied to both ECG and PPG signals. As it can be seen, the position of the dominant peak in frequency spectrums corresponds to the $\mathrm{HR}$ in $\mathrm{Hz}$ or beats $/ \mathrm{min}$. The spectrum of PPG is less scattered than ECG and is more convenient for HR extraction. The students measure the HR at different conditions, relaxed and during physical exercise and make a graph of its trend.
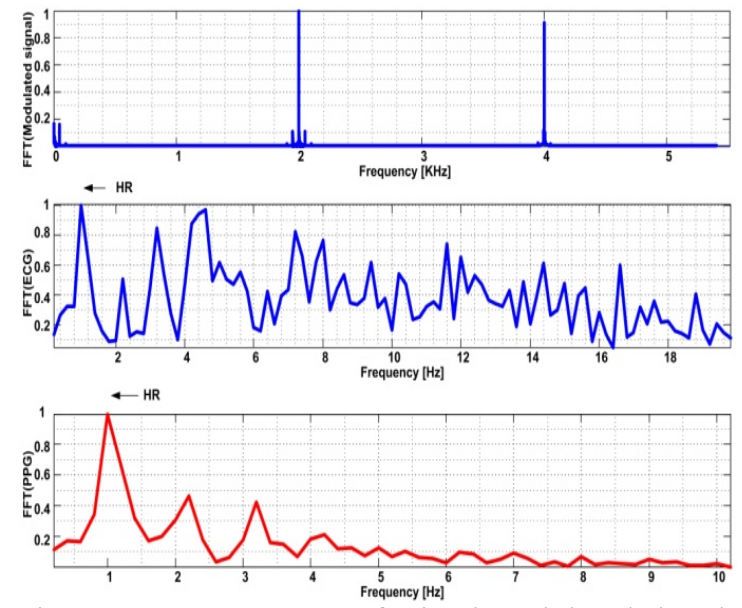

Figure 6. FFT spectrum of mixed modulated signal and PPG and ECG signals.

\subsection{Detection of QRS complexes and HRV analyze}

QRS complex is the most important segment in ECG signal and its shape, time of occurrence and frequency gives us valuable information about heart condition. Detection of QRS complexes is an important task in the analysis of ECG signals and starting point for many investigations and conclusions. Many methods exist on QRS detection, and an extensive review of the approaches proposed in the last decade can be found in [4]. One of the first and most effective is the Pan-Tompkins algorithm based on adaptive dual thresholding in which the decision rule utilizes a filtered signal and a version of the signal produced from a moving window integrator [5].

The students are introduced with steps of PanTompkins algorithm. Also, as a part of their homework they work on different algorithms, like ECG beat detection using filter banks, proposed by V. Afonso, W. Tompkins at all [6]. Initially, they test efficiency of the existing algorithms, off-line, by MIT-BIH database; and after that in real time using VI. Example of real-time QRS detection applying ECG filter banks is illustrated in Fig. 7. During QRS detection, the students note the effects of the algorithmic steps; low and high pass filtering, derivation, moving averaging, adaptive thresholds etc. They, also, clearly recognize the problem of QRS appropriations in real time conditions.

From ECG signal after implementation of QRS detectors they extract the vector of inter beat intervals (IBI) which gives the time distance between two successive R peaks, Fig. 8 (up). IBIs are associated with variation in heart rhythm and HRV which has a close relationship to sympathetic and parasympathetic nervous systems and is an important index to assess and monitor cardiovascular diseases or symptoms such as arrhythmia, coronary artery diseases, myocardial infarction, diseases, hypertension, etc [7]. HRV can be studied by different methods, but two are the most common: time domain to determine the deviation of successive R-R intervals; and spectral domain to determine the power spectral density of definitive frequency components of the ECG. The first step in the HRV analysis is the expression of successive R-R intervals in the record as a function of the time or the heartbeat number in the record, Fig. 8 (up). The second step is the conversion of the product of the first step, which is a tachogram, to HRV power spectrum by using FFT, Fig. 8 (down). The final step is the determination of the power spectrum density of certain 
frequency ranges in the HRV spectrum. As an example LP is the power density number for the low frequency range $(0.04-0.15 \mathrm{~Hz})$ that is generated mainly by sympathetic activity. HP is the power of the high frequency range $(0.15-0.40 \mathrm{~Hz})$ and is derived from vagal activity which is modulated by respiration. Their ratio is a good indicator of symphatetic-vagal balance. The students notice and discuss LP and HP concentrations from diagrams like in Fig. 8 (down).

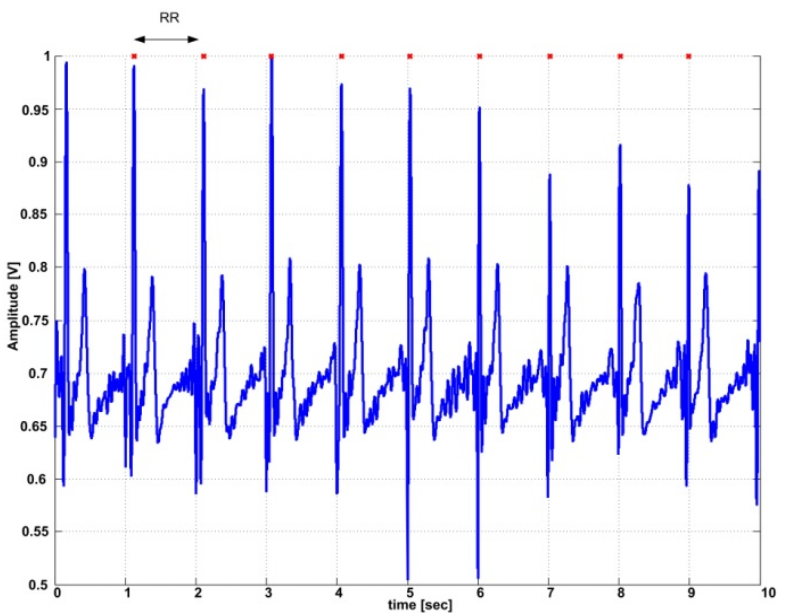

Figure 7. QRS detection, R-R intervals.
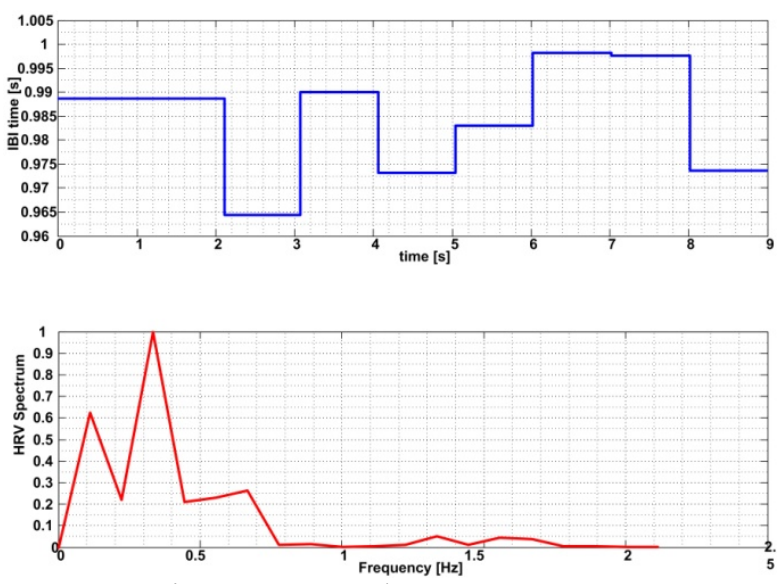

Figure 8. IBI and HRV spectrum.

\section{Conclusion}

The paper describes the development of a variety of classical biomedical experimental exercises by using standard tools such as amplifiers, modulators, microcontrollers, microphone input and MATLAB software. Signal acquisition is performed by sound card through microphone input which is a very convenient way. Multiple signals are transmitted through one cable using frequency multiplex technique. The exercises are developed by the fusion of the knowledge from various relevant disciplines and are intended to introduce students to fundamental concepts of biomedical experimentation, from the instrumentation and data acquisition requirements to subsequent data analysis techniques. A case study emphasizes the processing of PPG and ECG signals, but the same principle can be applied to other signals. Additionally, the approach can be successfully used in research purposes where virtual instruments should be use as a cheap and effective replacement for expensive instruments.

\section{References}

[1] M. Cano, E. Mena, R. Jaso, E. Palomar-Lever, J. De la Roca Chiapas, T. Córdova-Fraga, "Personal Device for Recording and Modulating the Electrical Activity generated by a Hearth through a PC Sound Input", International Journal of Bioelectromagnetism, Vol. 12, No. 2, pp. 85 -88, 2010.

[2] R. Stojanovic, D. Karadaglic, B. Asanin, O. Chizhova, "A Feasible Teaching Tool for Physiological Measurements", IFMBE Proceedings, Bamidis-Pallikarakis (Eds), Springer 2010, Vol. 29, pp. 959-963.

[3] K. W. Chan , K. Hung, Y. T. Zhang, "Noninvasive and cuffless measurements of blood pressure for telemedicine", Proceedings of the 23rd Annual International Conference of the IEEE, Volume 4, Issue , 2001 p.p. 3592 - 3593 vol.4.

[4] B. Kohler, C. Hennig and R. Orglmeister, "The principles of software QRS detection", IEEE Eng. Med. Biol. Mag., Vol. 21, pp. 42-57, 2002

[5] J. Pan and W. J. Tompkins, "A real-time QRS detection algorithm”, IEEE Trans. Biomed. Eng., Vol. 32, no. 3, pp. 230-236, 1985.

[6] V. Afonso, W. Tompkins, T. Nguyen, and S. Luo, "ECG beat detection using filter banks", IEEE Trans. Biomed. Eng., vol. 46, no. 2, pp. 192-202, 1999.

[7] Task Force of the European Society of Cardiology and the North American Society of Pacing and Electrophysiology. Heart rate variability: standards of measurement, physiological interpretation, and clinical use. Circulation, 1996, Vol. 93, pp. 1043-1065.

\section{Acknowledgment}

Some results of the project "New concept in development of WHC systems", financed by Montenegrin Ministry of Science, are incorporated into paper. We are thankful for their support. Also, great thanks to students Vladimir Popović, Jovan Kovačević, Boris Jovanović, Milan Rakojević for their significant contribution. 